\title{
LINEAMIENTOS METODOLÓGICOS PARA INCORPORAR LA EXPRESIÓN PLÁSTICA EN EL PLANEAMIENTO ESCOLAR
}

\author{
Julieta Castro Bonilla \\ Profesora - Escuela de formación Docente \\ Investigadora del Instituto de Investigación para el \\ Mejoramiento de la Educación Costarricense
}

\author{
Recibido 10-III-2004 • Aceptado 11-V-2004
}

"Todos nacemos con habilidades creadoras, pero aun así,
con lo universal que pueden ser estas cualidades,
también son frágiles y fácilmente destructibles".

Mimi Brodsky Chenfeld

\begin{abstract}
Resumen: El presente artículo se dirige especialmente a aquellos docentes que, sin poseer una formación idónea en el campo de la expresión plástica, desean incorporarla como parte de su metodología de trabajo. Conviene recordar que esta área no sólo se ofrece en algunas escuelas como una materia más dentro del curriculum escolar, sino también como un recurso didáctico que el docente puede utilizar como parte de su planeamiento cotidiano, por ello es fundamental su conocimiento y apreciación. Se espera que los lineamientos propuestos en el artículo ayuden al "maestro de grado" a desarrollar su propia sensibilidad, de manera que sus estudiantes puedan entrar en contacto con los contenidos presentes en las diversas áreas del curriculum escolar de manera lúdica, asimismo, le permita organizar y planificar lecciones agradables, de modo que los participantes en el proceso didáctico puedan disfrutar al máximo de la expresión plástica.
\end{abstract}

Palabras clave: Educación Primaria, Expresión Plástica, Lineamientos Metodológicos, Docentes, Estudiantes.

\section{Introducción}

El presente artículo se dirige especialmente a los maestros de primero y segundo ciclo de la Educación General Básica que, sin poseer una formación específica en el campo de las artes plásticas, desean incluir esta área, para reforzar, concretar y especialmente, permitirle a los estudiantes su interpretación y explicación de los conocimientos adquiridos mediante la expresión del lenguaje gráfico-plástico.

Para que lo expuesto se pueda concretar como parte del trabajo de aula, es necesario que el maestro comprenda la importancia de las Artes Plásticas, pues además de ser una materia impartida por docentes con una formación idónea en ésta área, se puede convertir en un recurso didáctico que le permite al alumno su integración a otras áreas del curriculum escolar. Conviene recordar que como responsable 


\begin{abstract}
The present article is addressed to teachers that wish to add to their work method the field of expression by fine arts, even when they do not possess an ideal training in that field. It must be remembered that this is not only offered in some schools as just another subject in the learning curriculum, but also as a learning resource that the teacher may use as part of its daily plan; therefore. its acknowledgement and appraisal as such is fundamental. It is expected that the suggested outlines in the article help the "grade teacher" to develop its own sensibility $y$, so that his or her students may establish a contact with the diverse contents of the different areas of their curriculum in a ludic way. It is also desirable that the teacher may be able to organize and plan enjoyable lessons, so that the children taking part in the learning process may savor the expression by fine arts to the fullest.
\end{abstract}

Key words: Elementary School, Expression by the Fine Arts, Methodological Outlines, Teachers, Students. de organizar el trabajo de aula, el docente tiene la delicada tarea de crear metodologías que se adapten a los estilos de aprendizaje de sus estudiantes, sin perder de vista los contextos en que se desenvuelven, por lo que debe planificar lecciones que propicien la convivencia escolar agradable y placentera.

\section{La expresión plástica, concepto e importancia para el niño}

Respecto al término "expresión plástica”, se considera relevante indicar que a este grupo expresivo corresponden básicamente las manifestaciones pictóricas, escultóricas y arquitectónicas, denominadas también artes visuales. No obstante, mi propósito en el artículo que presento es referirme al ámbito que abarca la "expresión plástica", o "artes plásticas", en el que se evidencia "la línea" como su principal medio expresivo.

En relación con el concepto "artes plásticas", Beuchat C. y otros (1994), indican que la línea como punto de partida de la expresión plástica puede ser tratada de distintos modos, pues ofrece infinidad de posibilidades para generar formas sobre superficies o en planos bidimensionales, ya sea mediante el dibujo, el grabado, la pintura o el mural. Por consiguiente, en la expresión plástica las formas se logran mediante líneas que se enriquecen con los siguientes elementos: colores, luces, sombras y texturas. Cabe destacar que la distribución u organización de esos elementos (de manera agradable o no), se logra mediante "la composición", la que resulta siempre atractiva si quien la realiza es un niño. En otras palabras, los elementos organizados y cargados de contenido simbólico encierran la personalidad de quien los expresa, ya que las características personales del autor se imprimen en el trabajo que realiza; por lo tanto, "la importancia que adquiere la ejercitación 
de la expresión plástica en el desarrollo de la imaginación es innegable si se considera que al dibujar, el niño representa y organiza imágenes estructuradas por él, a partir de conocimientos y formas de pensar reales e ideales, pero enriquecidas por su facultad de imaginar", (Beuchat C. y otros 1994, 84). La experiencia escolar demuestra que cuando el niño experimenta con diversos materiales y sobre espacios bidimensionales, su propósito primordial es el logro de diseños, pues le permiten su realización personal y provocan en él diversos estados de ánimo: sorpresa, alegría, asombro, aprobación, y también rechazo. Para que lo expuesto se pueda lograr de manera natural y espontánea, el estudiante debe tener acceso a diversos materiales, pues éstos harán posible la realización de productos de arte que le permiten su realización personal. A continuación hago referencia a esos materiales:

a. Soporte o superficie sobre la que se realiza la experiencia de arte, me refiero al papel, tela, cartón, pizarra, muros, otros.

b. Técnicas pictóricas, entre las que se pueden citar: témpera, pastel, acuarelas, lápices de colores, marcadores, pinturas preparadas, entre otras.

La combinación que se produce entre el soporte o superficie, las técnicas pictóricas y la aplicación de elementos expresivos (líneas, sombras, colores y texturas), le permitirán al estudiante su activa participación en procesos creativos que transmiten su personalidad, su capacidad expresiva, su grado de adquisición de conocimientos, su capacidad de percibir, de expresar emociones y sentimientos; así como sus experiencias personales e interpersonales, lo que contribuye al fortalecimiento de su desarrollo personal y social.

A continuación haré referencia a errores que según Alvarado A. y Murano G. (1990), cometen algunos de nuestros maestros, y quizás por desconocimiento los llevan a la práctica sin advertir sus consecuencias. Se mantiene la idea de que las Artes Plásticas es una disciplina exclusiva de quienes poseen habilidades y aptitudes para desempeñarse como artistas, por lo que con gran desacierto enseñan a dibujar artísticamente a sus estudiantes. Otro error es el de considerar que la tarea del docente de "expresión artística" se reduce a dejar el tema en manos de los alumnos, o a enseñarles una técnica o un determinado material para que trabajen sin orientación alguna. Para otros, este concepto se refiere a permitirle a sus alumnos que dibujen lo que se les ocurra, pues según ellos, dibujar de manera libre es sinónimo de expresión, y finalmente hay quienes se limitan a enseñar técnicas estimulando la copia o el calco de láminas, sin que se produzca ningún tipo de incentivación, o en otras situaciones, estimulando la reproducción de láminas sin la orientación didáctica o artística que se requiere.

Tomando en cuenta los enunciados anteriores, el docente interesado en aplicar la expresión plástica como un recurso didáctico, debe comprender que el arte para el niño escolar no se produce de manera mágica, ya que requiere de incentivos o recursos externos para que se produzca la motivación, ingrediente fundamental para el desarrollo de su imaginación; por lo que es necesario recordar que una de las tareas prioritarias de todo educador, es innovar constantemente su trabajo de aula, es producir en los estudiantes deseos de comunicar sus conocimientos, sus pensamientos, sus ideas, sus deseos, es comprender que la expresión plástica no sólo permite su manifestación como persona, sino el disfrute de lo que hace, sabiendo que las experiencias que lleva a la práctica con materiales y técnicas pictóricas fortalecen su creatividad y su personalidad, por lo tanto, le corresponde al maestro proporcionar una atmósfera agradable, que invite a la participación en actividades que expresen su capacidad cognitiva, afectiva, psicomotora y social. 


\section{La expresión plástica en el curriculum escolar}

Para un educador es fundamental reconocer que todas las áreas o asignaturas que conforman el curriculum escolar tienen su razón de ser, ya que cada una responde a necesidades específicas que el contexto y la sociedad le asignan. Sin embargo, cuando nos referimos a áreas con una marcada fundamentación humanista, como es el caso de las que conforman el grupo artístico (expresión: corporal, musical, plástica y dramática), la justificación no es tan clara, ya que generalmente estas áreas o materias de enseñanza van ligadas a la valoración que la misma sociedad les impone, y precisamente la expresión plástica se ha convertido en una de esas áreas que la sociedad valora con un bajo porcentaje. Con frecuencia el entorno familiar del estudiante no presenta una disposición de apertura a favor de la expresión artística, por lo que el escolar no siempre se encuentra motivado a aceptarla como una actividad que refleja su personalidad, a pesar de que esta área expresiva es del agrado de nuestros alumnos.

Es importante acotar que durante los últimos gobiernos, el Ministerio de Educación Pública ha impulsado de manera significativa políticas a favor de las áreas expresivas, a pesar de que cada vez se reducen las plazas de docentes que imparten este tipo de asignaturas.

No obstante lo anterior, con gran acierto algunos directores de instituciones de educación primaria, quizás quienes poseen un mayor grado de sensibilidad personal, permiten la creación de talleres en los que participan de manera voluntaria los docentes y estudiantes que se sienten atraídos hacia esta área expresiva. En otros casos, nos encontramos con maestros que poseen un afecto especial hacia el arte, pues valoran la importancia de este tipo de actividades en los niños y se dan a la tarea de planificar talleres de expresión dentro de su período lectivo, lo que conduce a una visión integradora del curriculum escolar.

Con gran acierto, la Escuela de Formación Docente de la Universidad de Costa Rica, ofrece a los estudiantes que ingresan al "Bachillerato en Educación Primaria", el curso Artes Plásticas aplicadas a la Educación, el cual brinda "la oportunidad de conocer la fundamentación teórica que le permite al futuro docente comprender y valorar la expresión artística como un medio creador y comunicativo en el desarrollo integral del niño. Se pretende que los futuros educadores conozcan las manifestaciones gráfico-plásticas del niño, según su edad e intereses. Ofrece además la oportunidad de desarrollar su propia sensibilidad y capacidad expresiva, mediante la experimentación con diversos materiales y técnicas artísticas de las áreas del dibujo, la pintura, la impresión, el modelado y la construcción" (Tomado de su descripción curricular, 2003). Como parte de la metodología que se aplica en el curso, los participantes se involucran de manera activa en las actividades que se programan. Por una parte, se estudian los fundamentos teóricos de las artes plásticas, por otra, se planifican talleres de expresión en los que se experimenta con variedad de soportes, técnicas y materiales. El curso se ofrece a una población heterogénea, por consiguiente, unos alumnos se entusiasman más que otros. Para quienes poseen habilidades y capacidades creativas más desarrolladas, el curso resulta interesante y es vivenciado de manera placentera. Quienes se consideran "poco aptos", y creen no poseer esa sensibilidad natural, también lo disfrutan, ya que la interacción grupal, el convivio, el respeto por la individualidad y el logro de cada participante es valorado de manera positiva. El curso cumple con las expectativas de los estudiantes pues permite su aplicación y transferencia a otros contextos según lo expresan ellos mismos. A pesar de que quienes lo reciben impartirán posteriormente las materias básicas del curriculum escolar: matemáticas, 
ciencias, estudios sociales y español, el propósito del mismo es que despierten en sus alumnos escolares interés hacia la expresión plástica. El curso enfatiza que, durante el período escolar, esta área expresiva se refiere principalmente a la comunicación espontánea y significativa que experimenta el niño, pues en este proceso creativo, éste selecciona aquellos elementos o cosas que se encuentran a su alrededor y las incorpora a los conocimientos que ya posee, para organizar, posteriormente, un nuevo conjunto que será representado a su manera, y de acuerdo con su propia interpretación, en este proceso se refleja su desarrollo cognitivo, social, afectivo, físico y estético.

Para que el docente pueda planificar de una manera realista su trabajo de aula, es importante que conozca las etapas evolutivas de sus estudiantes, ya que la organización de las actividades debe responder a sus intereses personales y sociales.

Para establecer la clasificación de las etapas evolutivas, se toman en cuenta aquellas características del dibujo infantil que surgen de manera espontánea en niños de la misma edad mental, en esta clasificación se desglosan los siguientes aspectos: el desarrollo del grafismo, la manera de distribuir el espacio, el diseño de las formas, el uso del color y los temas expresados. En el abordaje de este tema, quienes han profundizado en su estudio de una manera excepcional han sido Viktor Lowenfeld y Lambert Brittain, (1970), quienes han establecido la siguiente clasificación:

Primera etapa: Garabateo; sin control, controlado, con nombre; de 2 a 4 años.

Segunda etapa: Pre-esquemática; de 4 a 6 años.

Tercera etapa: Esquemática; de 6 a 9 años.

Cuarta etapa: Realismo; de 9 a 13 años.
Se considera importante indicar que las edades a las que corresponde cada etapa no deben tomarse de manera literal o "al pie de la letra", ya que varían de acuerdo con la estimulación que ha recibido el niño, el contexto socio cultural al que pertenece, así como el tipo de experiencias que haya tenido desde su nacimiento, por lo que el maestro debe aceptar las experiencias creativas de sus estudiantes sin poner objeciones.

Para que las actividades se puedan realizar de una manera natural, productiva y significativa, los docentes que atienden las áreas curriculares básicas, deben comprender la importancia que posee la planificación de experiencias artísticas como parte de su trabajo cotidiano, ya que permiten incursionar en nuevas formas de enseñar, y en consecuencia lograr mejores resultados en los aprendizajes de sus estudiantes. Asimismo, se debe tener presente que el contexto escolar y el extra escolar deben estar encaminados a facilitar los procesos de construcción del conocimiento, así como el desarrollo de habilidades, actitudes y aptitudes en sus estudiantes. A pesar de que gran parte de nuestras instituciones oficiales carecen de recursos didácticos y espacios exclusivos para el desarrollo de cualidades creativas y expresivas en estudiantes, es importante mencionar que al trabajar con niños, el maestro es quizás el ingrediente más importante, ya que éste se debe convertir en un guía y en un facilitador del aprendizaje, para lo que es fundamental el respeto a la personalidad de cada estudiante.

A. Alvarado y G. Murano, (1990), mencionan algunas de las actitudes que el maestro debe tener presente al organizar su trabajo de aula:

- Debe trabajar amorosamente con sus estudiantes.

- $\quad$ Ser apasionado de la expresión plástica.

- $\quad$ Fomentar en el alumno el pensamiento divergente, ayudándolo a buscar 
respuestas para la satisfacción de él mismo.

- Respetar por igual todas las ideas procedentes de los estudiantes.

- Disfrutar con los alumnos de sus descubrimientos.

- $\quad$ Favorecer el proceso más que el resultado, ayudándole a cada uno a autoevaluarse.

- Sensibilizar al alumno frente a la naturaleza y frente a su condición humana.

- Atender la problemática de cada uno en particular, pero sin perder de vista al grupo.

- $\quad$ Promover temas de interés.

- $\quad$ Ser flexible con los materiales y técnicas. El alumno debe conocer variedad para que pueda representar lo que desee.

- No olvidar que, para fomentar cambios de actitud en los alumnos, debemos empezar por cambiar nosotros.

Las actitudes docentes mencionadas, son ingredientes que se consideran básicas para la planificación de actividades metodológicas, pues permiten interacciones grupales que facilitan el estudio de contenidos curriculares de una manera lúdica.

\section{La incorporación de las artes plásticas al currículum escolar, lineamientos metodológicos}

Muchos de los niños que asisten a nuestras instituciones de educación primaria del sistema oficial, no tienen la oportunidad de disfrutar activamente de la asignatura denominada "Artes Plásticas", pues ésta es impartida especialmente en instituciones particulares, y bajo la responsabilidad y guía de docentes con una formación académica idónea en las áreas: artística y pedagógica. Como lo mencionara anteriormente, reconociendo que la expresión plástica es disfrutada plenamente por nuestros niños, le corresponde al maestro de grado planificar actividades que permitan la expresión de sus conocimientos mediante vivencias que se puedan relacionar con su contexto escolar y familiar.

Como parte de sus tareas, el educador debe conducir al niño desde su mundo de lo concreto hasta el pensamiento abstracto "cuando los pequeños llegan a preescolar se encuentran en su primera etapa de desarrollo, y en este nivel las actividades de expresión plástica ofrecen la oportunidad de experimentar una y otra vez con diversos materiales y pasar de una manera sana y equilibrada por el proceso de las primeras representaciones cognoscitivas y los preconceptos, hasta el razonamiento operatorio, al que llegará en el período escolar". (G. Sefchovich y G. Waisburd, 1999, 76).

De acuerdo con lo expuesto, le corresponde al docente la responsabilidad de seleccionar aquellos tópicos que sean realmente interesantes y susceptibles de convertirse en aprendizajes significativos. Con frecuencia nos encontramos con niños que no comprenden temas de áreas como matemáticas, ciencias y otras, debido a que se les exige apoyar el aprendizaje únicamente con la memoria y no con la percepción, con la experiencia y con la lógica, pues son los caminos que conducen de forma natural a la abstracción, sin atropellar los procesos de crecimiento y maduración del niño. (G. Sefchovich y G. Waisburd, 1999, 77).

Antes de referirme a los lineamientos metodológicos que el docente puede incorporar como parte de su trabajo, deseo recodar que la programación de actividades se 
debe organizar tomando en cuenta las siguientes facetas: 1- la determinación de los objetivos; 2- la clasificación de los contenidos, incluyendo los dominios que los estudiantes deben alcanzar: cognitivos, afectivos y psicomotores; 3- la determinación de los procedimientos metodológicos o la planificación de las situaciones de aprendizaje, entre las que se citan las actividades de iniciación o incentivadoras, de desarrollo y de culminación; 4- determinación de los procesos evaluativos.

Los lineamientos metodológicos que se exponen en el presente artículo, tienen el propósito de acercar a los docentes que imparten las asignaturas básicas a la expresión plástica, para lo que recomiendo la incorporación de las "etapas", de observación, de asociación y de expresión de conocimientos, a los procedimientos metodológicos o actividades: de iniciación o incentivadoras, de desarrollo y de conclusión o de cierre. Es importante indicar que si el maestro participa plenamente en las actividades que él mismo desarrolla, probablemente contagiará con su entusiasmo a sus estudiantes, lo que facilitará la integración de experiencias académicas y artísticas, desde una visión integral de ser humano.

A continuación me referiré a cada una de las "etapas" mencionadas, aunque el maestro puede incluir otras de acuerdo con su propia investigación, su experiencia y creatividad, sin olvidar las edades e intereses de sus estudiantes, las diferentes maneras de aprender y los contextos en donde viven sus propias realidades.

Etapa de observación: Es importante tener presente que la expresión plástica es uno de esos fenómenos cuyo análisis y estudio debe realizarse teniendo como soporte la imagen. El arte infantil no es una idea que se expresa de manera abstracta, sino concreta, por lo que la experimentación, el empleo de materiales y técnicas pictóricas, se convierten en un medio para expresar de manera placentera sus ideas, sentimientos y emociones. Para que esta etapa resulte significativa, le corresponde al docente organizar ambientes agradables dentro y fuera del aula, que inviten a la imaginación y a la inventiva, asimismo, debe dedicar un período de su lección a incentivar y a estimular los sentidos por ser considerados la base del aprendizaje. Es importante mencionar que son los alumnos quienes se motivan, pues se trata de un proceso interno que se produce en cada persona de acuerdo con el grado de estimulación externa. En otras palabras, la capacidad creadora necesita de los estímulos externos para ponerse en funcionamiento, por lo que conviene la planificación de actividades que involucren: lectura de poesías, cantos, narración y dramatización de poemas, observación de piezas de arte y fotografías, comentario de vídeos, de documentales y noticias referidas al contexto, finalmente, la recreación de hechos y acontecimientos científicos e históricos permiten el logro de una motivación que facilita la construcción de contenidos de manera significativa. Es conveniente recordar la importancia que posee el contacto directo con seres vivos, o con materiales naturales o artificiales como: metales, maderas, arcilla, arena y otros.

Esta etapa se puede correlacionar con las actividades denominadas "introductorias o incentivadoras", por lo que le corresponde al docente:

- Desarrollar situaciones de aprendizaje que estimulen los sentidos en los alumnos, ya que éstos se consideran fundamentales en la experiencia pedagógica y artística.

Etapa de asociación: se correlaciona con las actividades "de desarrollo", en ésta los ejercicios de asociación son aún más significativos en la medida en que los estudiantes se encuentran motivados y avanzan en su desarrollo cognitivo, 
personal y emocional, ya que podrán adentrarse en el estudio e investigación de temas que permiten establecer asociaciones entre los diversos contenidos del curriculum escolar y la expresión artística. El maestro debe recordar que la planificación de actividades metodológicas grupales e individuales (dentro o fuera del aula) es considerada vital para el estudiante, pues permite el logro de la socialización e individualización, principios básicos para la formación de su personalidad. El maestro que imparte las materias básicas del curriculum escolar, debe comprender que los contenidos no deben ser estudiados o investigados de manera independiente o aislada, por lo tanto, tendrá la delicada tarea de seleccionar de manera coherente los temas y subtemas de los programas de estudio, afines entre sí, con el propósito de organizar metodologías que permitan la construcción significativa de esos contenidos por parte de sus alumnos. Cuando el docente considera que ha habido conocimiento y comprensión de los temas estudiados, la expresión plástica se puede convertir en un recurso didáctico, ya que permite reforzar lo aprendido desde una perspectiva pictórica, pues al ser vivenciada y recreada la experiencia académica, el aprendizaje resultará realmente significativo. Se espera que las reflexiones expuestas, de alguna manera le permitan al docente crear conciencia sobre la importancia de mantenerse actualizado, no solo en cuanto a su formación básica, sino en el campo de la apreciación y la expresión artística, lo que le permitirá incursionar de una manera lúdica en la planificación de actividades.

\section{Etapa de expresión de conoci-} mientos: es conveniente relacionar esta etapa con las actividades de culminación o período durante el cual los estudiantes expresan o comunican lo aprendido o investigado pero desde un planteamiento personal. Esta etapa favorece la explicación y la interpretación de lo asimilado mediante diversos lenguajes: pintura, dibujo, acuarela, murales, otros, y de manera individual o grupal. Es importante que el docente comprenda la importancia de la participación estudiantil durante todas las etapas mencionadas, lo que permitirá una fácil integración de temas o áreas curriculares que podrá transferir a otros contextos. Las actividades grupales, la participación en conferencias y trabajos investigativos y otros, le permiten al estudiante enriquecerse de los procesos de enseñanza preparados por quien facilita el aprendizaje, por lo que conviene:

- Estimular a los alumnos para que vivencien sus conocimientos, sus deseos y emociones, tomando en cuenta su realidad sociocultural, de esta manera se podrá propiciar una apertura hacia la expresión plástica de manera natural.

- Valorar la expresión artística de los alumnos como piezas únicas, lo que permite que sea respetada por ellos mismos, por sus compañeros y familiares.

- Conocer las particularidades que presentan los dibujos realizados que los niños, para lo que se debe tomar en cuenta la edad cronológica y el nivel que cursan.

Como se mencionara anteriormente, para que las artes plásticas se puedan disfrutar plenamente, el docente puede lograr su integración a otras áreas del curriculum escolar permitiendo que el alumno exprese de manera pictórica, natural y placentera sus aprendizajes, posterior a las etapas mencionadas.

Lineamientos metodológicos en la programación de actividades de expresión plástica: se trata de la programación de actividades que permiten el logro de 
aprendizajes. Surgen de la necesidad de sistematizar el trabajo de aula de manera que su correcta aplicación trae como resultado la construcción de conocimientos y la adquisición de destrezas en el estudiante. Se debe tener presente que los resultados se logran a corto, a mediano y a largo plazo, pues la creatividad y el acto de expresar son procesos que no deben ser forzados, surgen en cada estudiante de manera espontánea, según sus experiencias, vivencias, necesidades, aprendizajes y motivación. Los lineamientos metodológicos que a continuación se indican, se generan de cuatro fuentes: desde mi participación en un trabajo investigativo relacionado con la expresión artística, como profesora del curso mencionado anteriormente, de mi experiencia como profesora en cursos de capacitación a docentes de educación primaria, en donde el contexto de aula se convierte en centros de experiencias, y finalmente de la literatura leída. Como lo indicara en el apartado anterior, la programación de actividades debe tener el propósito de facilitarle al estudiante una integración de contenidos que le permita construir con facilidad sus propios conocimientos, y por lo tanto su propio aprendizaje, por lo que le corresponde al maestro la selección de contenidos programáticos de manera coherente y tomando como punto de partida los objetivos específicos de la lección.

Como lo expuse anteriormente, las experiencias que el maestro acumula como parte de su trabajo de aula resultan fundamentales para la programación de actividades interesantes y atractivas. Algunas veces la metodología puede ser sugerida por textos, la que debe ser adecuada a los diversos contextos de aula; otras veces es organizada por el grupo de compañeros que comparten el mismo nivel, lo que resulta interesante y beneficioso ya que la discusión y reflexión favorece los procesos pedagógicos, en otras situaciones se puede tomar en cuenta la participación y experiencia de los alumnos, lo que también resulta enriquecedor. Lo importante no es cómo surge la programación de las actividades y etapas mencionadas, sino cómo los participantes se enriquecen de las propuestas que el docente prepara con entusiasmo. Los lineamientos que a continuación se indican, son recursos que el educador puede integrar a su trabajo cotidiano, pues contribuyen al desarrollo de los valores estéticos y ofrecen una visión de integralidad.

- $\quad$ La ambientación del aula, lo estético y atractivo de la presentación de los materiales serán elementos incentivadores que despiertan el interés hacia el trabajo por realizar.

- $\quad$ En la programación, el maestro debe tener presente la importancia de brindar variedad de oportunidades para todos los participantes, sin olvidar las diferencias individuales.

- $\quad$ El maestro deberá descubrir, recuperar, desarrollar y aplicar su potencial creativo, asimismo, definir claramente la normativa en cuanto al respeto, orden, limpieza, y otros, principios indispensables durante el desarrollo del taller de expresión de arte.

- La incorporación de las "etapas" de: observación, asociación y expresión de conocimientos a las actividades de introducción o incentivadoras, de desarrollo y de conclusión, aseguran de alguna manera el logro de los objetivos propuestos.

Los lineamientos indicados contribuyen a la planificación de un trabajo interesante y productivo en el que se procura la estimulación del pensamiento divergente, considerado clave para la seguridad y el bienestar de cada estudiante, y en donde las características humanas como la curiosidad, la intuición, la capacidad de analizar y sintetizar, la flexibilidad y otras, se estimulan 
por medio de la expresión artística. (Vargas Ana Isabel, 1986).

Como parte de la fundamentación teórica del artículo, se considera importante acotar que las artes plásticas presentan dos modalidades de trabajo que no se deben ofrecer de manera aislada: la expresión y la apreciación, ambas tienen en común una estructura basada en los siguientes elementos: la línea, la luz, los colores, la textura, y la distribución armónica de esos elementos en espacios o superficies planas, en volúmenes y en construcciones espaciales que permiten el logro de formas. Mediante la expresión, el niño comunica sus conocimientos y estados de ánimo manipulando los elementos mencionados: líneas, luces, colores, texturas, que los distribuye y organiza de manera armónica de acuerdo con su forma de ser y tomando en cuenta la técnica y los materiales empleados. La apreciación permite que el niño emita su criterio personal, para lo que debe observar, mirar, ver, tocar y oler sus propias realizaciones, las de sus compañeros o alguna pintura de un artista reconocido. Ambas modalidades se deben considerar como parte de las experiencias artísticas que el docente planifica dentro o fuera del aula. (Ronderos, María Elena y otros. 2000).

Al organizar actividades que impliquen la correlación de contenidos, el docente debe seleccionar cuidadosamente el tema o contenidos por estudiar, por lo que es necesario que conozca a profundidad los contenidos de los programas emanados por el Ministerio de Educación, pues son los que forman parte de su programación diaria, así como la determinación del contexto en el que desarrollará su trabajo. Para que el nuevo material de aprendizaje resulte significativo para el estudiante, es conveniente la formulación de preguntas que permitan reaccionar, cuestionarse, indagar, investigar, y también dudar, de esta manera el estudiante intervendrá con entusiasmo en el proceso creativo, pues se sentirá atraído y compenetrado en la actividad. Es fundamental recordar que todas las respuestas emanadas por los estudiantes son aceptadas, aún las incorrectas, pues lo valioso es la participación natural y espontánea de todos los integrantes del grupo.

Como lo indicara anteriormente, uno de los propósitos del presente artículo es que una vez clasificados y desglosados los contenidos de manera secuencial, coherente, y de lo general a lo particular, el docente logre correlacionarlos con otras áreas curriculares y otros temas de interés como los denominados "ejes curriculares", lo que favorece una concepción integradora del curriculum que le permite al niño recrear y vivenciar los conocimientos adquiridos desde una perspectiva gráfico-plástica.

Al considerar que la naturaleza es una fuente inagotable de inspiración, pues permite establecer relaciones entre temas y contenidos cualquiera que sea el área, el maestro debe estimular la percepción de sus elementos, ya que nos transmiten sensaciones que podemos expresar mediante colores, texturas, luces, sombras, volúmenes, entre otros.

Para que se pueda gestar un trabajo de aula de calidad, el maestro debe crear ambientes que conduzcan a la inventiva, a la exploración, a la imaginación, en donde las habilidades, destrezas, sentimientos $\mathrm{y}$ emociones de los estudiantes contribuyan a la concreción de sus aprendizajes. En gran medida, en nuestras instituciones de educación primaria, los contenidos curriculares son impartidos de manera aislada, creando brechas que provocan situaciones artificiales y confusas de parte del que aprende, por lo tanto, el maestro no debe variar el origen y la realidad científica de los temas que conforman los diversos planes de estudio, por lo que debe reconocer que en cualquier experiencia artística, el entusiasmo de los niños depende en gran medida del grado de motivación en que se encuentren, por lo que conviene brindar a todos los estudiantes oportunidades para que externen sus criterios, sus puntos de vista y sus opiniones personales. 


\section{Conclusión}

De acuerdo con el presente artículo, las artes plásticas como actividad expresiva se pueden convertir en un recurso que contribuye al desarrollo de las áreas cognitiva, afectiva y psicomotora de nuestros estudiantes; por lo que le corresponde al docente que imparte lecciones en educación primaria, organizar ambientes de aprendizaje en los que se puedan vivenciar y concretar los aprendizajes de manera significativa.

Las reflexiones que indico a manera de sugerencias, podrían formar parte de la programación de actividades pedagógicas y artísticas que el docente planifica:

- $\quad$ En la educación primaria, las artes plásticas deben ser consideradas un medio expresivo natural y espontáneo, por lo que no es necesario enseñar a dibujar artísticamente a los estudiantes; lo importante es brindarles los suficientes soportes o superficies, variadas técnicas pictóricas, para que con la ayuda de los elementos expresivos, pueda expresar sus creencias y experiencias de manera libre y espontánea.

- La experiencia de arte no sucede al azar, por lo que es necesario preparar al estudiante de manera afectiva y mediante actividades incentivadoras que despierten los sentidos por considerarse éstos la base para el aprendizaje.

- La expresión artística es quizás la única disciplina que realmente se concentra en el desarrollo de experiencias sensoriales, ya que éstas se encuentran llenas de ricas texturas, de variadas y caprichosas formas y de la profusión del color, y tanto el estudiante como el docente deben estar capacitados para encontrar placer y alegría en estas experiencias.
- Las artes creadoras deben ser consideradas un importante recurso en nuestro sistema educativo, ya que impulsan el pensamiento divergente, en el que no existe respuesta correcta, por lo que se deben aceptar todas las soluciones posibles a los problemas que el maestro plantea.

- La capacidad creadora necesita alimentarse de una especial atmósfera, por lo que el docente debe guiarla para que ésta refuerce el pensamiento creador, y de esta manera los niños puedan desarrollar su imaginación y sus ideas novedosas sin censura de parte de los adultos.

En el planteamiento y organización de actividades creativas como parte del trabajo de aula, el maestro debe tener presente que no se trata de imponer normas, sino de permitirle a sus estudiantes desarrollar la capacidad para interpretar, discriminar, elegir, seleccionar y expresarse con libertad, ya que la expresión no solo responde al reflejo de su personalidad, sino de su contexto escolar y familiar, por lo que se le debe permitir a cada estudiante la oportunidad de vivenciar e interpretar de manera libre y espontánea los contenidos desarrollados durante el período lectivo.

Para que el trabajo con los estudiantes se pueda llevar a cabo de manera efectiva y productiva, es necesario que el docente practique y desarrolle su propia sensibilidad en relación con el medio, de manera que al entrar en contacto con las manifestaciones artísticas de sus alumnos, pueda valorar mejor el trabajo de cada uno.

Las reflexiones y sugerencias que presento, se dirigen especialmente a los maestros de educación primaria, que sin poseer estudios específicos en el área de formación artística, desean incorporar la expresión plástica como parte de su trabajo cotidiano, ya que permite una concepción 
dinámica de las asignaturas que conforman el currículum escolar, pues se generan acciones que le permiten al niño el desarrollo de una personalidad integral, lo que contribuye a formar ciudadanos capaces de crear cosas nuevas y no simplemente repetir lo que otros han hecho, ciudadanos que sean capaces de investigar, de descubrir, de cometer errores pero también de corregirlos, de transferir los conocimientos y aprendizajes a otras situaciones. Espero que el presente artículo sea un estímulo para la búsqueda de estrategias que potencien el disfrute del aprendizaje en los alumnos, sabiendo que cada situación en clase debe tratarse como una experiencia única y irrepetible, que requiere de soluciones específicas dependiendo del contexto, de los niveles de desarrollo de los estudiantes, de los temas y de los objetivos propuestos. Para concluir, deseo incorporar una cita que encierra de alguna manera el planteamiento teórico expuesto “...en la medida en que a una persona se le amplíen los conocimientos y experiencias, su mundo se va enriqueciendo y al mismo tiempo su capacidad de transmitir o expresarse se enriquece también, ya que tiene mayor cantidad de vivencias $\mathrm{y}$, por ende tiene una mayor y mejor posibilidad de relacionar y combinar los elementos en su interior, lo cual se traducirá en respuestas más ricas, más propias y más creativas" (Beucaht Cecilia y otros. 1993, p. 65).

\section{Referencias bibliográficas}

Acerete Dora M. Objetivos y didáctica de la educación plástica. Guía para el maestro de grado. Argentina. Editorial Kapelusz, 1984.
Albarado Ana y Murano, Gabriela. El taller de plástica en la escuela. Teoría y práctica. Argentina: Editorial Troquel, 1990.

Beucaht Cecilia y otros. Desarrollo de la expresión integrada: Club Cli-Clopips. Chile: Editorial Andrés Bello, 1993.

Herrera Marvin. Enfoque curricular del programa de estudios de Artes Plásticas. Ministerio de Educación Pública. San José, Costa Rica, 1991.

Lowenfeld Victor y Britain W. Lambert. Desarrollo de la Capacidad Creadora. Argentina. Editorial Kapelusz, 1970.

Pacheco Julia y Pacheco Mary Luz. Construyendo Imaginarios. Talleres creativos. Colombia. Cooperativa Editorial Magisterio, 1998.

Sefchovich Galia y Waisburd. Hacia una pedagogía de la creatividad. Expresión plástica. 2a a Edición. México. Editorial Trillas. 1987.

Ronderos María Elena y otros. Educación Artística. Lineamientos curriculares. Áreas obligatorias y fundamentales. República de Colombia, Ministerio de Educación Nacional. Cooperativa Editorial Magisterio, 2000.

Vargas D. Ana Isabel. Técnicas para el Desarrollo de la expresión y el Movimiento en la Escuela Primaria o Básica. Módulo 7. UNESCO/CAP. San José Costa Rica, 1986. Litografía Lil. S.A. (San José, Costa Rica). 